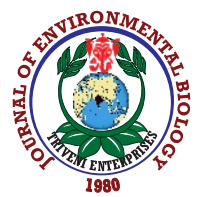

\title{
Investigating the applications of Chlorella vulgaris in agriculture and nanosilver production
}

\author{
M.F. Al dayel', N.A. El Semary ${ }^{* 1,2}$, K. Al Amer $^{3}$ and K.M. Al Ali ${ }^{1}$ \\ 'Department of Biological Sciences, College of Science, King Faisal University, Al-Ahsa-31982, Kingdom of Saudi Arabia \\ ${ }^{2}$ Department of Botany and Microbiology, Faculty of Science, Helwan University, Cairo-11795, Egypt \\ ${ }^{3}$ Department of Chemistry, College of Science, King Faisal University, Al-Ahsa-31982, Saudi Arabia \\ *Corresponding Author Email : nelsemary@kfu.edu.sa
}

\section{Abstract}

Aim: To investigate the dual beneficial effect of $C$. vulgaris as biofertilizer and antimicrobial agent in the form of nano particles.

Methodology: The antibacterial effect of aqueous extract was investigated by diffusion disk bioassay against five pathogenic bacterial strains. The plant growth promoting effect was investigated by performing germination tests on three types of seeds and measuring their morphometric growth parameters. The silver nanoparticles biosynthetic ability of alga was investigated by incubating with silver nitrate solution. The biogenic nanosilver was tested against multidrug-resistant Staphylococcus aereus(MRSA).

Results: The antibacterial effect of the extract, despite being derived from a miniscule biomass, was detected against all pathogenic strains. The inhibition zones ranged from 0.7 to $1 \mathrm{~cm}$. In addition, the aqueous extract was effective bio-fertiliser that resulted in a significant increase in germination percentage and growth parameters of Eruca sativa, Lepidium sativum and Vigna radiata seedlings. Furthermore, the silver nanoparticles were observed to form both intra- and extracellularly. The UV -visible spectrum of nanoparticles showed a characteristic broad peak at $421-425 \mathrm{~nm}$, while IR showed the presence of characteristic signal at $3297 \mathrm{~cm}^{-1}$. The nanosilver particles were effective antimicrobial agents against multi-drug resistant MRSA.

Interpretation: The results emphasize the multiple potential of $C$. vulgaris, which indicates its great potential if grown on a large scale for mass production. The present research also sheds some light on the potential of microflora inhabiting this rather underexplored part of the world.

Key words: Antibacterial, Bio-fertilizer, Chlorella vulgaris, Silver nanoparticle

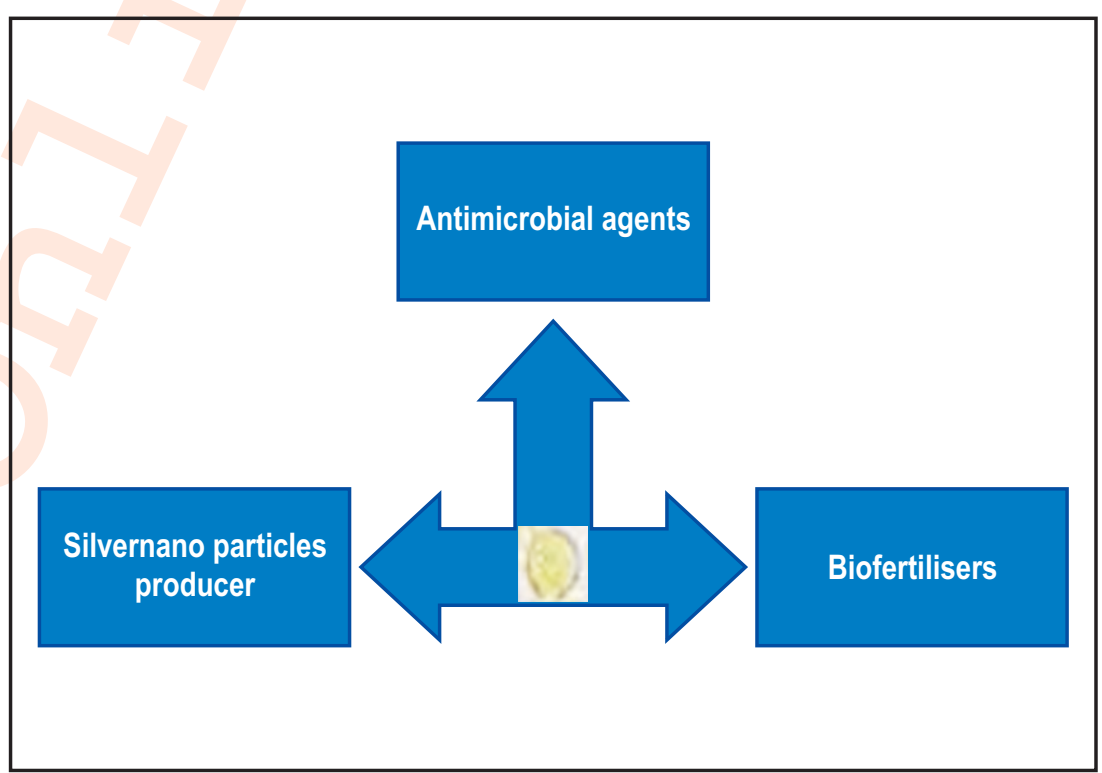

How to cite : Al Dayel, M. F., N. A. El Semary, K. Al Amer and K.M. Al Ali: Investigating the applications of Chlorella vulgaris in agriculture and nanosilver production. J. Environ. Biol., 41, 1099-1104 (2020). 


\section{Introduction}

Chlorella vulgaris is a green microalga that belongs to Order: Chlorellales, Family: Chlorellaceae (Bejerinick, 1890). It is widely distributed in both freshwater or marine ecosystem (Hodač et al., 2016). Cells of Chlorella species are small spherical or ellipsoidal whose chloroplast is cup-shaped lying in a parietal position. This alga is considered as a rich source of phytohormones (Tarakhovskaya et al., 2007) and essential nutrients. It is estimated to contain about $6 \%$ protein, $7.5 \%$ fat, and $18 \%$ carbohydrates. The protein of this alga contain essential amino acids, high percentage of beta-carotene and chlorophyll (Krienitz et al., 2018), vitamin A, thiamin (B1), riboflavin (B2), pyroxene (B6), niacin and cyanocobalamin (B12), C, E, K, minerals such as magnesium, potassium, phosphorus, copper, zinc, iodine, cobalt, calcium and iron and digestive enzymes (Sawant and Mane, 2018). All these attributes indicate high nutritional value of this alga. However, its use as a source of liquid bio-fertilizer (Ronga et al., 2019) with sparse reviews such as that of Priyadarshani and Rath (2012). (Faheed and Abd El Fattah, 2008; Bumandalai and Tserendulam. 2017). Ineterstingly, Chlorella extract has previously showed antibacterial activities (Acurio et al., 2018). Therefore, the beneficial rote of aqueous extract of $C$. vulgaris both as biofertilizer and as antibacterial agent against Bacillus sp., Streptococcus sp., Staphylococcus aureus and $E$ coli was investigated. Streptococcus sp. bacterium is found in the digestive system of humans and animals and are widely spread in plants and dairy products. Bacillus $\mathrm{sp}$. is a rod-shaped bacterium that plays an important role in the corruption of a number of foods and in food poisoning (Omardien et al., 2018). Esherichia coli which lives mainly in the digestive system human and animal are also present in soil. Its presence in food is indicative of faecal contamination (Malar et al., 2018). Lastly, Staphylococcus aureus is a Gram-positive bacterium with several strains being multidrug-resistant. It is also the most common cause of food poisoning (Ondusko and Nolt, 2018). These pathogenic bacteria are of different characteristics and quite difficult to find one antimicrobial agent that has broad-spectrum activity against all in addition to having plant growth-promoting activities. This is a necessity nowadays as there is a surge for searching for eco-friendly fertilizers that replace harmful chemical fertilizers. In addition, if these biofertlisers have antimicrobial affect, they can reduce the use of harmful pesticides. Therefore, in order to address its protective ability against multidrugresistant bacterial strain, the ability of nanosilver particles biosynthesis by this alga was investigated and the antibacterial action of biogenic nanosilver particles was tested against multidrug resistant Staphylococcus aureus (MRSA). Chlorella vulgaris has been reported to produce nanosilver (Soleimani and HabibiPirkoohi, 2017). Skulberg (2000) pointed out the need to exploit microalgae from rather underexplored areas for their applicable potential as they possess unique metabolites and bioactive compounds of wide array of uses and impact. Keeping in view the above, the present study was carried out to investigate the application of Chlorella vulgaris in agriculture and nanoparticles biosynthesis.

\section{Materials and Methods}

Collection and identification of samples : The algal sample was collected in a sterile sampler from an irrigation canal supplied by groundwater in the east of Al-Ahsa Governorate. Light microscopic examination was performed to determine the types of algae present. Bold's medium was used to culture microscopic freshwater algae. After successive agar-streaking trials followed by purification, the green alga Chlorella vulgaris was identified as verified by microscopic examination.

Preparation of aqueous algal extract: The aqueous extractwas prepared from $0.3 \mathrm{~g}$ fresh weight of Chlorella vulgaris biomass that was homogenized and extracted in $50 \mathrm{ml}$ of sterile distilled water. To test the effect of aqueous Chlorella vulgaris extract on germination of seeds of different plants, ten seeds with replicates of Vigna radiata, Eruca sativa and Lepidium sativum were washed and sterilised using $6 \%$ (v/v) bleach for few minutes, then washed thoroughly with sterile water several times and soaked in the aqueous extract for a day. Then the seeds were planted on a sterile wet cotton wool and observed for their germination for a week. Germination percentage, radical and plumule length were recorded for the treatments and compared with control.

Antibacterial bioassay : The antibacterial potency of aqueous extract obtained from Chlorella vulgaris, was assessed against pathogenic bacterial strains namely, Escherichia coli, Staphylococcus aureus, Streptococcus sp. and Bacillus sp. that were freshly sub-cultured prior to assay. Sensitivity of pathogenic strains to the extract was assayed by modified Kirby Bauer Disk Diffusion Susceptibility method (Bauer et al., 1966). Sterilizedpaper discs (6 $\mathrm{mm}$ in diameter) were saturated with $30 \mu \mathrm{l}$ of extracts for antibacterial tests. Discs were dried and placed on the surface of inoculated nutrient agar medium with bacterial suspensions prepared in physiological saline $\left(0.85 \mathrm{~g} \mathrm{NaCl}^{100 \mathrm{ml}^{-}}\right.$ ${ }^{1}$ distilled water). Plates were kept for $2 \mathrm{hr}$ at $4{ }^{\circ} \mathrm{C}$ to ensure diffusion of bioactive material, after that the plates were incubated at $37^{\circ} \mathrm{C}$. Control discs containing $30 \mu \mathrm{l}$ of sterilized distilled water were left to dry, then used as negative control whereas positive control sets included discs of antibiotic Chloramphenicol. Plates were incubated for $24 \mathrm{hr}$ and diameter of inhibition zones $(\mathrm{mm})$ were measured in triplicates and the average standard deviation was recorded (EI Semary, 2012). The antimicrobial bioassay was performed in a single experiment using nanosilver nanoparticles produced by Chlorella vulgaris, of low concentration $5 \mathrm{ug} \mathrm{ml}^{-1}$, as antimicrobial agent against multidrug-resistant strain of Methicillin-resistant staphylococcus aureus (MRSA).

Biosynthesis of silver nanoparticles by Chlorella cells: A stock solution of silver nitrate (Sigma-Aldrich, USA) of $1 \mathrm{M}$ concentration was prepared and used in study. The solution was 
Table 1 : Growth parameters (in percentage) of untreated (control) and treated seeds with aqueous extract of Chlorella vulgaris

\begin{tabular}{llll}
\hline Growth parameters & Seeds & Control & Chlorella vulgaris extract \\
\hline Germination percentage & Lepidium sativum & $100 \pm 0$ & $100 \pm 0^{\mathrm{a}}$ \\
& Eruca sativa & $100 \pm 0$ & $100 \pm 0^{\mathrm{a}}$ \\
& Vigna radiata & $100 \pm 0$ & $100 \pm 0^{\mathrm{a}}$ \\
Radicle length & Lepidium sativum & $0.99 \pm 0.3$ & $1.31 \pm 0.3^{\mathrm{b}}$ \\
& Eruca sativa & $1.03 \pm 0.4$ & $2.01 \pm 0.1^{\mathrm{b}}$ \\
Plumule length & Vigna radiata & $2.76 \pm 0.3$ & $3.73 \pm 0.3^{\mathrm{b}}$ \\
& Lepidium sativum & $0.67 \pm 0.3$ & $0.93 \pm 0.2^{\mathrm{b}}$ \\
& Eruca sativa & $0.88 \pm 0.3$ & $1.31 \pm 0.2^{\mathrm{b}}$ \\
& Vigna radiata & $0.65 \pm 0.2$ & $0.97 \pm 0.3^{\mathrm{b}}$ \\
\hline
\end{tabular}

${ }^{\mathrm{a}}$ Insignificantat $p<0.05 ;{ }^{\mathrm{b}}$ Significant at $p<0.05$ confidence

kept in dark bottle. Chlorella vulgaris biomass was collected by centrifugation $(6000 \mathrm{rpm})$ and thoroughly washed with distilled water and centrifuged again. The biomass was incubated with silver nitrate solution for two months, both algal biomass and outer solution turned brown and were analyzed for silver nanoparticles production. The cells of Chlorella vulgaris were homogenized and the resultant homogenate was centrifuged for $30 \mathrm{~min}$ at $6000 \mathrm{rpm}$ for complete precipitation of all cell debris. Supernatant was collected and filtrated using sterilized $0.2 \mu \mathrm{m}$ millipore filter membrane. Filtrate was collected and used for the analysis of silver nanoparticles (AgNPs). Biogenic silver nanoparticles were analyzed and characterized by the following analyses.

UV-visible spectroscopic (UV-vis) analysis: The initial characterization was carried out by a UV-vis spectrophotometer (Genesys10S UV-visible double beam spectrophotometer). The spectrum was recorded at room temperature using $200 \mu$ test volumes with $1 \mathrm{~cm}$ path length quartz cuvette, in a wavelength range of $200 \mathrm{~nm}$ to $800 \mathrm{~nm}$. Nanoparticle solution was diluted 5 times with deionized water to avoid errors due to high optical density of the solution.

Dynamic light scattering analysis: Dynamic light scattering (DLS) was studied with Dual Scattering Particle Size Analyzer (cilas, Nano DS). It was used to determine the average particle size and distribution of AgNps. Each sample was interpreted in triplet at $25^{\circ} \mathrm{C}$ with scattering angle of $135^{\circ} \mathrm{C}$. Deionized water was used as medium disperses.

Infrared spectrometer analysis of AgNPs : Characterization of AgNPs An Fourier-Transform infrared spectrometer (FT-IR, Agilent Cory 630, Agilent Technologies, USA) was used to analyze the chemical composition of nano particles.

Statistical analyses : Statistical significance of results was tested by Analysis of Variance (ANOVA) at $p<0.05$. One way Anova test was performed using Minitab version 13.

\section{Results and Discussion}

Chlorella sp. is a widespread green alga found all over the world. Its wide presence in diverse habitat is an indicative of its
Table 2 : Antibacterial activity (zone of inhibition) of Chlorella vulgaris aqueous extract

\begin{tabular}{ll}
\hline Bacterial species & Inhibition zone \\
\hline Staphylococcus aureus & $0.8 \pm 0.1$ \\
Streptococcussp. & $0.7 \pm 0.1$ \\
Bacillussp. & $1.0 \pm 0.2$ \\
Methicillin-resistant staphylococcus aureus & $0.7 \pm 0.1$ \\
E.coli & $0.9 \pm 0.1$ \\
\hline
\end{tabular}

adaptive metabolic capabilities (Hodač et al., 2016). Chlorella sp. is rich in many bioactive compounds such as minerals, vitamins, growth factor and is used in healing wounds and as a food (Jensen, 1987). The use of Chlorella sp. for enhancing seed growth was reported by Faheed and Abd El Fattah (2008) who used Chlorella treatment on seeds of Lactuca sativa which significantly increased the seed growth. Addition of microalga to the culture medium or soil significantly increased the fresh and dry weight of seedlings. They attributed this enhancing effect to the presence of high amount of macronutrients, micronutrients and amino acids in the microalgal treatment. Al-Shakankery et al. (2014) showed that Chlorella vulgaris treatment significantly enhanced the growth of maize plants both quanti and qualitatively mainly due to the presence of many growth-promoting compounds. In the present study, C. vulgaris showed enhancing effect on different plant seeds. The germination rate of Vigna radiata seeds with Chlorella vulgaris aqueous extract was faster than that of control where the germination percent reached $90 \%$ on first day while that of control was only $70 \%$. Both reached $100 \%$ germination percentage on the second day. The average length of radical and plumule were significantly greater than that of control (Table 1).

On the other hand, the germination rate of Eruca sativa seeds treated with $C$. vulgaris extract was equal to that of the control on all days. However, there was a different trend with regard to plumule and radical length. The highest radical length was obtained for the treatment with aqueous extract of Chlorella. Nonetheless, both radicle and plumule length were significantly greater than control. 

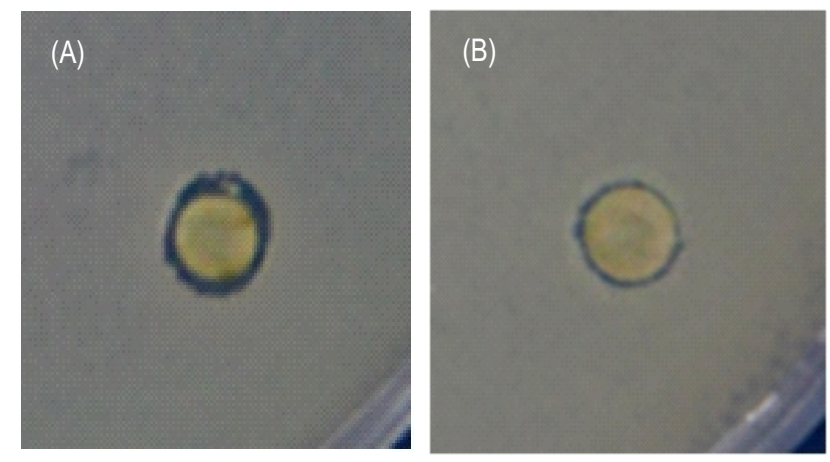

Fig. 1: Inhibition zone of aqueous extract of Chlorella vulgaris against (A) Bacillus sp. and (B) Methicillin-resistant staphylococcus aureus.

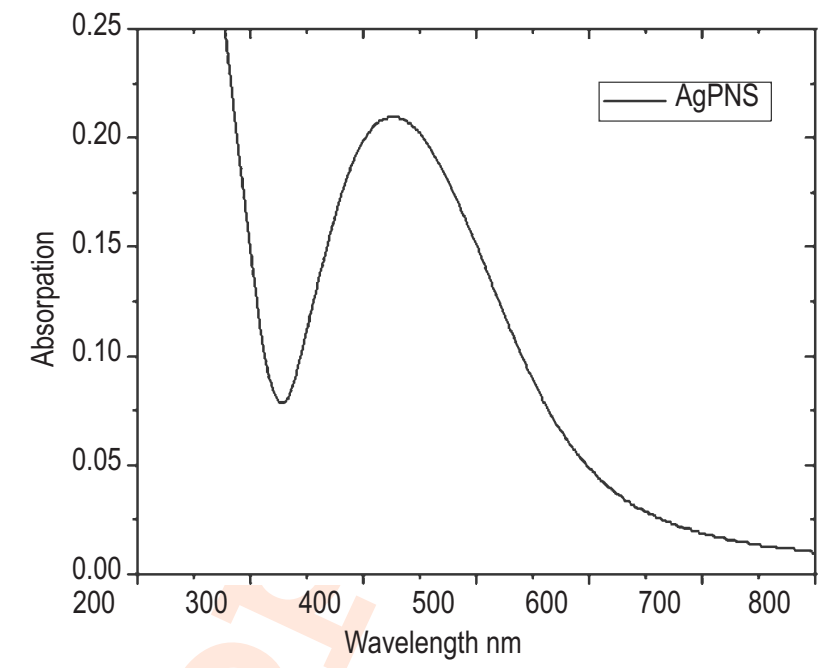

Fig. 2 : UV spectra for AgNPs.

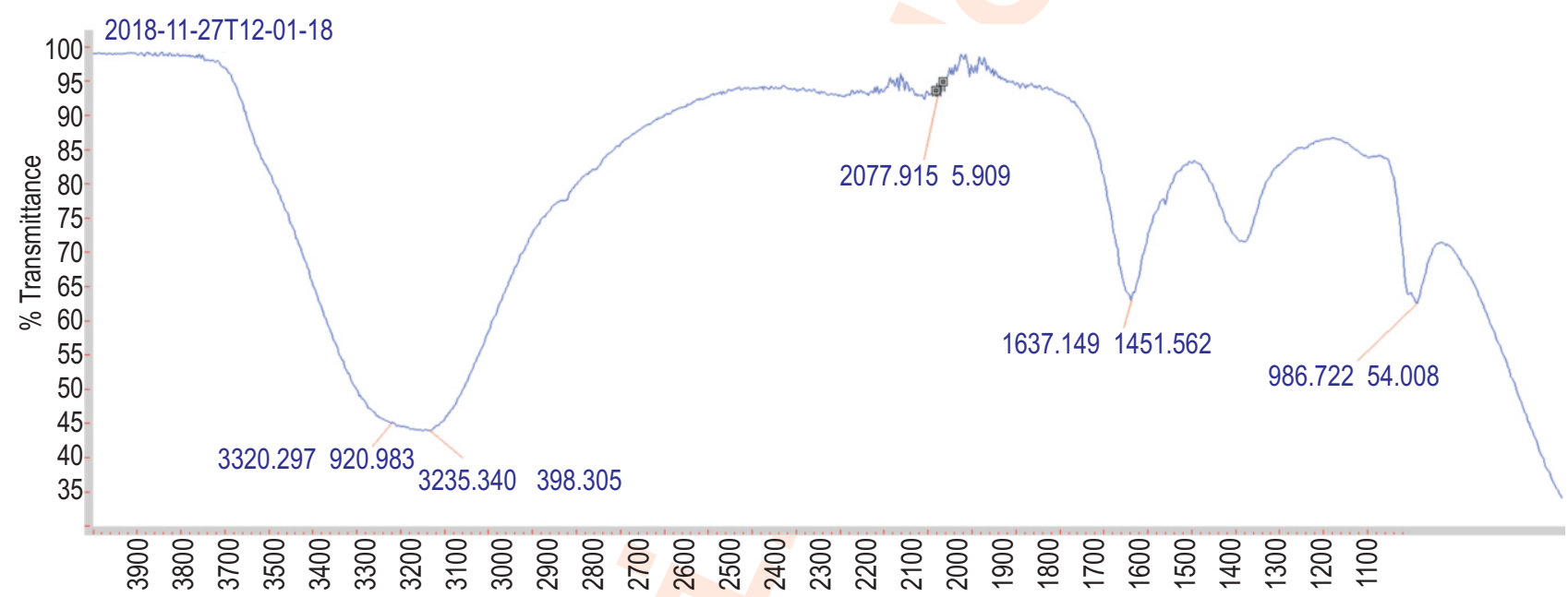

Wave number

Fig. 3 : FTIR spectrum for nanosilver particles.

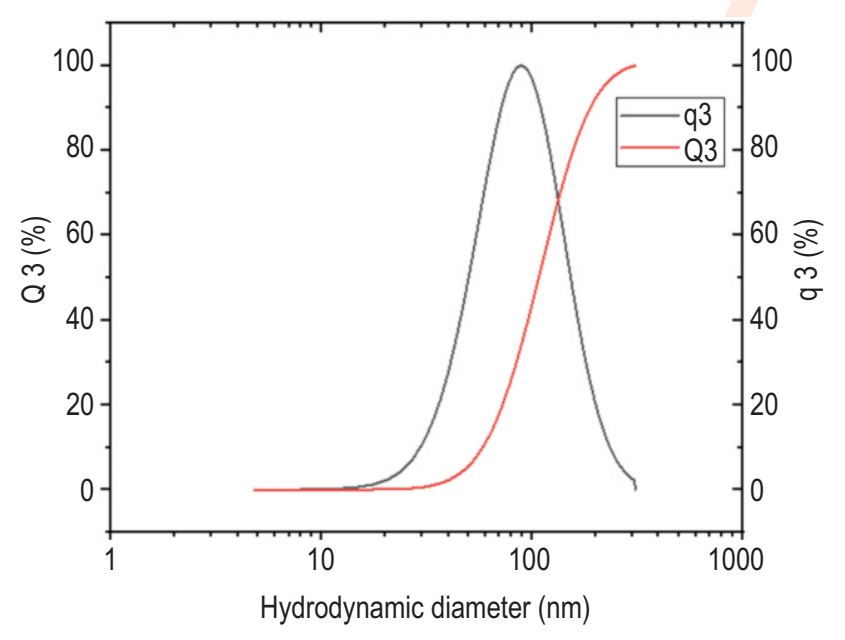

Fig. 4 : Distribution particle size by Dynamic light scattering for AgNPs.
Finally, the germination rate of Lepidium sativum seeds after treatment with extract was faster than control. The seeds showed $100 \%$ germination on the first day whereas the control showed only $90 \%$. They both reached $100 \%$ on the second day (Table 1) Similar to the other two types of seedlings, the plumule and radicle length were significantly greater than control. Regarding the nature of plant growth promoting substances, Stirk et al. (2013 a, b) attributed this to the presence of several growth promoting hormones such as indole acetic acid, cytokinins, gibberllins and brassinosteroids. The latter accumulates in the nucleus and is responsible for significant induction of stem elongation. The fact that the aqueous extract of C. vulgaris had an enhancing effect on seeds reflect its agrobeneficial impact.

Regarding Chlorella extract as antimicrobial agent, the presence of several bioactive compounds such as flavanoids, 
tannin, phenolic compounds, terpenes, glycosides, saponins has been reported (Shabudeen et al., 2015). Acurio et al. (2016) reported the extraction of chlorellin as antimicrobial compound, which has antibacterial effect comparable to ampicillin and oxacillin. Moreover, Shabudeen et al. (2015) highlighted the importance of aqueous extract of Chlorella vulgaris rich in phenols antibacterial against different Gram positive and negative bacteria which is in complete accordance with our results. (Dantas et al., 2015). Some of the phenols that Chlorella sp. contain include simple phenols, derivatives of benzoic acid and cinnamic acid, coumarins and flavonoids. In accordance with aforementioned reports, the aqueous extract derived from a very small biomass ( $0.3 \mathrm{~g}$ fresh weight) showed a broad-spectrum antibacterial effect against all tested bacterial pathogens, both Gram positive and negative (Table 2). The greatest inhibition zone was obtained for Bacillus sp. (Fig. 1A) whereas the smallest was that Methicillin-resistant staphylococcus aureus (Fig. 1B). However, low concentration silver of nanoparticles $\left(5 \mathrm{ug} \mathrm{ml}^{-1}\right)$ showed antimicrobial agent against the multidrug resistant strain of Methicillin-resistant staphylococcus aureus. The inhibition zone was $1.2 \mathrm{~cm}$.

Nano-silver particles were successfully synthesized both intra and extra-cellularly from green Chlorella vulgaris cells. The formation of AgNPs was confirmed by UV- vis absorbance measurement. UV-Vis spectra of AgNPs are presented (Fig. 2) in the range of $200-800 \mathrm{~nm}$. The colloidal solution of synthesized AgNPs showed a strong specific peak for synthesized AgNPs was obtained at $425 \mathrm{~nm}$ (Fig. 2). The presence of dark brown color confirmed the production of AgNPs, and the increased absorption refers to increased concentration-This is in accordance with the characteristic bands in the UV-Visible spectrometeric analysis indicative of silver nanoparticles which showed plasmon resonance in the range of 410-450nm (Yasin et al., 2016). In addition, FTIR confirmed their biosynthesis by Chlorella. The FTIR spectra of the AgNps. (Fig. 3) clearly showed that nanosilver particles were present at $3297 \mathrm{~cm}^{-1}$ overlapping with $\mathrm{OH}$ signal (Verma et al., 2015). At $1637 \mathrm{~cm}^{-1}$ there was a C-H stretching vibration of aromatic ring, and at $1040 \mathrm{~cm}^{-1}$ there was C-O-C stretching vibration of aromatic ether. The main components of Chlorella vulgaris is cellulose, which contain phenolic hydroxyl, benzene ring, double bond, - $\mathrm{O}$-, and other groups. All these functional groups contain lone electron pairs. When nano-scale micro emulsion is formed in the aqueous phase, these functional groups were dispersed on the surface of emulsion, showing a strong adsorption capacity.

Dynamic light scattering technique was used to measure the light intensity reflected by particles in solution. DLS analysis (Fig. 4) showed a wide range of particle size distribution varying AgNPs from 55.9 to $106.9 \mathrm{~nm}$ with average value of $87.8 \mathrm{~nm}$. The size of most particles was around $90 \mathrm{~nm}$. The use of this technique is based on certain facts. The use of reflected light vary over time as particles change their position due to Brownian motion. The rate of this process depends on the hydrodynamic diameter of particles. Therefore, by measuring the rate of change of reflected light, the size of suspended particles can be estimated. The majority of particles were $90 \mathrm{~nm}$ in size with some proportion being higher or lower than that. This size is quite favorable for different application of silver nanoparticles as it has a large surface area which means higher interaction surface than that of larger particles. In addition, the cost-effectiveness and pollution-free of biologically-synthesized nanoparticles make them more plausible than those synthesized using harmful and expensive chemical and physical synthesis methods (Mandal et al., 2006). The proposed mechanism of nanosilver biosynthesis is that metals are trapped on the surface of microalgae via carboxylate group, the metal ions are reduced by reducatse elemental form via the action of reductase enzymes (Kannan and Subbalaxmi, 2011). Finally, the use of produced biogenic nanosilver as antibacterial agent against multi-drug resistant strain MRSA is highly effective. It is in accordance with the reports of Marambio-Jones (2010). The reports emphasize the bacteriocidal effect of silver nanoparticles of three different sizes and its broad-spectrum activity against both Gram-positive and Gram-negative bacteria, including multi-resistant strains. This can be eco-friendly strategy for cost-effective bacterial control.

These promising results only indicate that this alga if grown on mass scale can be exploited in several ways. However, the cost of cultivation and maintenance of cultures on inexpensive growth media are issues of concern as the cost of microalga is high production (Mata et al., 2010). Regard the use of wastewater or recycled water from hydroponic cultures such as that of tomatoes were proposed (Zhang et al., 2017). This waste water is loaded with nutrients and represent cost-effective system for algal production andoxygen evolved in photosynthesis can be used in tomatoes respiration and growth which is a positive indication of success of hydroponic algal/tomato coproduction. Overall, this is the first report, to the best of our knowledge from Saudi Arabia that explores the multiple potentials of local green alga Chlorella vulgaris which showed promising potentials although the tested biomass was miniscule. This indicates great potential this alga, if used on mass scale. The study highlights the biotechnological potentials of microbial isolates from underexplored localities due to unique metabolism and activities.

\section{Acknowledgment}

The authors would like to express their deepest gratitude and acknowledge the financial support of Deanship of scientific Research, King Faisal University, Al- Ahsa, Nasher track, grant number 186125. 


\section{References}

Acurio, L.P., D.M. Salazar, A.F. Valencia, D.R. Robalino, A.C. Barona, F.C. Alvarez and C.A. Rodriguez: Antimicrobial potential of Chlorella alga isolated from stacked waters of the Andean Region of Ecuador. Environ. Earth Sci., 15, 012040 (2018).

Al-Shakankery, F.M., R.A. Hamouda and M.M. Ammar: The promotive effect of different concentrations of marine algae as biofertilizers on growth and yield of maize (Zea mays L.) plants. J. Chem. Biol. Phys. Sci., 4, 43201-43211 (2014)

Bauer, A.W., W.M. Kirby, J.C. Sherris and M. Turck: Antibiotic susceptibility testing by a standardized single disk method. Am. J. Clin. Pathol., 45, 493-496 (1966).

Beyerinck [Beijerinck], M.W.: Culturversuche mit Zoochlorellen, Lichenengonidien und anderen niederen Algen. Botanische Zeitung (Cultivation experiments with Zoochlorella lichenengonidia and other lower algae). Botanische Zeitung, 47, 725-739, 741-754, 757-768, 781-785(1890).

Bumandalai, O. and D. Tserendulam: Effect of Chlorella as a biofertilizer on germination of wheat and barely grains. Proceed Mongolian Acad. Sci., 56. 26. 10.5564/pmas.v56i4.839. (2017).

Dantas, D., R. Costa', M.G. Carneiro-da-Cunha', A.O. Galvez, A.R. Drummond and R.S. Bezerra: Bioproduction, antimicrobial and antioxidant activities of compounds from Chlorella vulgaris. Res. Rev. J. Bot. Sci., 4, 12-18 (2015)

El Semary, N.A.: The characterisation of bioactive compounds from an Egyptian Leptolyngbya sp. strain. Ann. Microbiol., 62, 55-59 (2012)

Faheed, F. and Z. Abd El Fattah: Effect of Chlorella vulgaris as biofertilizer on growth parameters and metabolic aspects of lettuce plant. J.Agric. Soc. Sci., 4, 165-169 (2008)

Hodač, L., C. Hallmann, K. Spitzer, J. Elster, F. Fabhauer, N. Brinkmann, D. Lepka, V. Diwan and T. Friedl: Widespread green algae Chlorella and Stichococcus exhibit polar-temperate and tropicaltemperate biogeography. FEMS Microbiol Ecol., 92, fiw122 (2016).

Jensen, B.: Chlorella: Gem of the Orient: Escondido, California: Jensen Publication (1987).

Kannan, N. and S. Subbalaxmi: Biogenesis of nanoparticles: A current perspective. Rev. Adv. Mater. Sci., 27, 99-114 (2011).

Krienitz, L., V.A. Huss and C. Bock: Chlorella: 125 years of the green survivalist. Cell Press, 1240, 1-3 (2018).

Malar, A.S., M. Sekar, K. Porteen, R. Narayanan, A. Elang, A. Kumar, M. Sowmiy and T. Devi. Screening of E. coli for its antimicrobial susceptibility pattern in milk and dairy products in Chennai, India. Int. J. Chem. Stud., 6, 35-37 (2018).

Mandal, D., M.E. Bolander, D. Mukhopadhyay, G. Sarkar and P. Mukherjee: The use of microorganisms for the formation of metal nanoparticles and their application. Appl. Microbiol. Biotechnol., $69,485-492(2006)$
Marambio-Jones C. and E. Hoek: A review of the antibacterial effects of silver nano materials and potential implications for human health and the environment. J. Nanopart. Res., 12, 1531-1551 (2010).

Mata, T.M., A.A. Martins and N.S. Caetano: Microalgae for biodiesel production and other applications: A review. Renew. Sustain. Energy Rev., 14, 217-232 (2010).

Omardien, S., A.T. Beek, N. Vischer, R. Montijn, F. Schuren and F. Brul: Evaluating novel synthetic compounds active against Bacillus subtilis and Bacillus cereus spores using live imaging with SporeTrackerX. Sci. Rep., 8, p. 9128 (2018)

Ondusko, D.S. and D. Nolt: Staphylococcus aureus. Ped. Rev., 39, 1-27 (2018)

Priyadarshani, I. and B. Rath: Commercial and industrial applications of micro algae. Areview. J. Algal Biomass UtIn., 3, 89-100 (2012).

Ronga, D., E. Biazzi, K. Parati, D. Carminati, E. Carminati and A. Taya: A review: Microalgal biostimulants and biofertilisers in crop productions. Agronomy, 9, 192 (2019)

Sawant, S.S. and V.K. Mane: Nutritional profile. antioxidant, antimicrobial potential and bioactives profile of Chlorella emersonii KJ725233. Asian J. Pharm. Clin. Res., 11, 220-225 (2018).

Shabudeen, S., A. Arasu and I. Ponnuswamy: The uses of Chlorella vulgaris as antimicrobial agent and as a diet: The presence of bioactive compounds which caters the vitamins, minerals in general. Int. J. Bio-Sci. Bio-Technol., 7, 185-190 (2015).

Skulberg, O.: Microalgae as a source of bioactive molecules Experience from cyanophyte research. J. Appl. Phycol., 12, 341$348(2000)$

Soleimani, M. and M. Habibi-Pirkoohi: Biosynthesis of silver nanoparticles using Chlorella vulgaris and evaluation of antibacterial efficacy against Staphylococcus aureus. Avicenna J. Med. Biotechnol., 9, 120-125 (2017).

Stirk, W.A., V. Ördög, O. Novák, J. Rolèik, M. Strnad, P. Bálint and J. Staden: Auxin and cytokinin relationships in 24 microalgal strains. J. Phycol., 49, 459-467 (2013 a).

Stirk, W.A., P. Bálint, D. Tarkowská, O. Novák, M. Strnad, V. Ördög and J. van Staden: Hormone profiles in microalgae: Gibberellins and brassinosteroids. Plant Physiol. Biochem., 70, 348-353 (2013b).

Tarakhovskaya, E.R., Yu.I. Maslov and M. Shishova: Phytohormones in algae. Russ. J. Plant Physiol., 54, 163-170 (2007).

Verma, K., S.H. Hasan and R.M. Banik: Swift green synthesis of silver nanoparticles using aqueous extract of Tamarindus indica leaves and evaluation of its antimicrobial potential. Int. J. Innov. Res. Sci. Eng. Technol., 4, 11182-11190 (2015)

Yasin, S. and Y. Liu: Biosynthesis of silver nanoparticles by bamboo leaves extract and their antimicrobial activity. J. Fiber Bioeng. Inform., 6, 77-84 (2013).

Zhang, J., X. Wang and Q. Zhou: Co-cultivation of Chlorella spp. and tomato in a hydroponic system. Biomass Bioenerg, 97, 132-138 (2017). 